\title{
Variación altitudinal en caracteres morfológicos de Pinus patula en el estado de Oaxaca (México) y su uso en la zonificación
}

\author{
Altitudinal variation in morphological traits of Pinus patula populations \\ from Oaxaca state, Mexico, and its use in the zoning
}

\author{
Héctor Viveros-Viveros a*, Alfredo R Camarillo-Luna ${ }^{\text {b, }}$ \\ Cuauhtémoc Sáenz-Romero ${ }^{\mathrm{b}}$, Armando Aparicio-Rentería ${ }^{\mathrm{a}}$
}

\footnotetext{
*Autor para correspondencia: a Universidad Veracruzana, Instituto de Investigaciones Forestales, Parque Ecológico “El Haya”, Colonia Benito Juárez, Xalapa, Veracruz, México. C.P. 91070, tel.: 2288421700 ext. 13964, heviveros@hotmail.com

${ }^{\mathrm{b}}$ Universidad Michoacana de San Nicolás de Hidalgo, Instituto de Investigaciones Agropecuarias y Forestales (IIAF-UMSNH), Morelia, Michoacán, México.
}

\begin{abstract}
SUMMARY
Populations of forest species with a distribution across altitudinal gradients tend to differ in morphology, allowing zoning for conducting seed movement. The pattern of variation is not known to many Mexican pine species. The altitudinal pattern of morphological variation was explored in needles, cones and seeds of 13 Pinus patula populations sampled from the Native Indian Community forest of Ixtlán de Juárez, Oaxaca, Mexico, along an altitudinal gradient (from 2,450 to 3,050 m a.s.l); needles and cones being collected every $50 \mathrm{~m}$ of altitudinal step. Significant differences among populations and among trees within populations were detected for all the evaluated traits. The correlation was positive between the altitude of origin of populations and keel width, cone width and leaf-sheaths length; whereas it was negative between needle length and altitude of origin of populations. Using the phenotypic variation of the needle length we suggest a provisional delineation of two altitudinal seed zones (zone I: 2,150 to 2,600 m; zone II: 2,600 to 3,050 m), as a guideline to decide seed movements in reforestation programs. Altitudinal zones obtained in this work have wider altitudinal range per zone (meaning a more liberal seed movement) than the one obtained from twenty four-months-old seedling height growing in a provenance test, and show that it is possible to make provisional altitudinal zoning based on patterns of phenotypic variation across environmental gradients.
\end{abstract}

Key words: altitudinal variation, provisional seeds zones, morphological traits, Pinus patula.

\section{RESUMEN}

Las poblaciones de especies forestales con distribución a través de gradientes altitudinales tienden a diferenciarse en sus caracteres morfológicos, lo que determina zonificaciones para el movimiento de semillas. El patrón de variación es desconocido para muchas especies de pinos mexicanos. Se exploró el patrón de variación morfológica en acículas, conos y semillas de 13 poblaciones de Pinus patula, muestreadas en bosques de la comunidad indígena de Ixtlán de Juárez, Oaxaca, México, a lo largo de un gradiente altitudinal (2.450 a $3.050 \mathrm{~m}$ s.n.m.), cada $50 \mathrm{~m}$ de diferencia altitudinal. Se encontró variación significativa entre poblaciones y entre árboles dentro de poblaciones para todas las características evaluadas. La correlación entre la altitud de las poblaciones y el ancho de quilla, el grueso del cono y la longitud de la vaina de la acícula fue positiva, mientras que entre la longitud de acícula y la elevación fue negativa. Según la variación fenotípica de la longitud de acícula, se sugiere la delimitación provisional de dos zonas semilleras altitudinales (zona I: 2.150 a 2.600 m; zona II: 2.600 a 3.050 m), como criterio para decidir el movimiento de semillas en programas de reforestación. La zonificación altitudinal obtenida en el presente trabajo tiene mayor amplitud altitudinal por cada zona (lo que significa movimiento más libre de las semillas), que el obtenido a partir del crecimiento en altura de las plantas a los 24 meses de edad en ensayo de procedencias y muestra cómo realizar zonificaciones preliminares según el patrón de variación fenotípica en gradientes ambientales.

Palabras clave: variación altitudinal, zonas semilleras provisionales, caracteres morfológicos, Pinus patula.

\section{INTRODUCCIÓN}

Pinus patula Schiede ex Schltdl. et Cham. es uno de los pinos mexicanos con mayor potencial productivo, debido a que es de rápido crecimiento, tiene buena conformación de copa y poda natural, su fuste es recto, y su madera es de calidad, al estar relativamente libre de nudos y presentar bajo contenido de resinas (Nyoka 2002). Por esta razón se ha plantado extensamente como especie exótica en lugares ubicados en el sur de África (Malawi, Mozambique, Sudáfrica, Suazilandia, Zambia y Zimbabue), en el este de África (Burundi, Etiopia, Kenia, Madagascar, Ruanda, Tanzania y Uganda), en el oeste y centro de África (Camerún, Nigeria y República Democrática del Congo [Zaire]), en Asia (China e India), en Oceanía (Australia, Fiji, Nueva Zelanda, Papua Nueva Guinea), y en el Caribe y Sudamé- 
rica (Argentina, Brasil, Colombia, Ecuador, Jamaica y Venezuela) (Nyoka 2002). La distribución natural altitudinal de P. patula oscila entre 1.600 y 3.100 m s.n.m. (Nyoka 2002). Este intervalo altitudinal tan amplio debe favorecer la diferenciación genética entre poblaciones.

Diversas poblaciones de coníferas que crecen a lo largo de gradientes altitudinales se adaptan a las diversas condiciones ambientales, por lo que se diferencian genéticamente (Rehfeldt 1991). Por ejemplo, las poblaciones de coníferas ubicadas a menor altitud sobre el nivel del mar, tienen un mayor potencial de crecimiento por estar genéticamente condicionadas a aprovechar al máximo las condiciones favorables de temperatura. En contraste, los individuos de poblaciones de mayor altitud están condicionados a tener un menor crecimiento, con lo que logran evadir los daños por heladas tardías en la primavera y heladas tempranas en el otoño (Rehfeldt 1991).

Lo anterior obliga al establecimiento de zonificaciones altitudinales para el movimiento de semillas basadas en la diferenciación genética de caracteres cuantitativos (Rehfeldt 1991, Sáenz-Romero 2004, Sáenz-Romero et al. 2006). La zonificación consiste en dividir la amplitud en que se distribuye naturalmente una especie en una región, en zonas o bandas altitudinales. La división se basa en encontrar experimentalmente la diferencia altitudinal mínima significativa que hace que dos o más poblaciones sean genéticamente diferentes, como resultado de la evaluación de algún carácter cuantitativo, como por ejemplo la altura de la planta o la resistencia a bajas temperaturas (Sáenz-Romero 2004). En México, actualmente se cuenta con la zonificación altitudinal para Pinus oocarpa Schiede (Sáenz-Romero et al. 2006), Pinus devoniana Lindl. (sinonimia Pinus michoacana Martínez) (Sáenz-Romero y Tapia-Olivares 2008), Pinus pseudostrobus Lindl. (SáenzRomero et al. 2012) y Pinus hartwegii Lindl. (Viveros-Viveros et al. 2009) en el estado de Michoacán, y Pinus patula Schltdl. et Cham. (Ruíz-Talonia 2010) en el estado de Oaxaca, con base en la variación genética del crecimiento en altura de la planta a los 24 meses de edad.

Las zonificaciones altitudinales, junto con las guías para el movimiento de semilla, se pueden utilizar para establecer con mayor probabilidad de éxito reforestaciones para restauración ecológica y plantaciones comerciales; así como para establecer medidas de conservación de las especies estudiadas (Rehfeldt et al. 2001, Sáenz-Romero et al. 2006, Viveros-Viveros et al. 2009), debido a que facilitan el acoplamiento entre las plantas y las condiciones ambientales de los sitios donde se establecerán las reforestaciones.

México es el país con mayor diversidad de especies del género Pinus, con aproximadamente 49 taxa, lo que representa cerca de la mitad de las especies conocidas de este género en el mundo (Styles 1993). Sin embargo, esta diversidad hace más compleja la realización de zonificaciones altitudinales para la mayoría de las especies de pinos en México. Zonificaciones como las descritas requieren de colectar semillas, producir planta en vivero, establecer, mantener y evaluar ensayos de procedencias, idealmente en varios sitios experimentales con condiciones ambientales contrastantes. Una alternativa temporal es la realización de zonificaciones provisionales para las poblaciones de cada especie a partir de la variación fenotípica altitudinal de caracteres, principalmente con valor adaptativo. Para México, Uribe-Salas et al. (2008) realizaron una zonificación para el encino blanco (Quercus rugosa Née) a lo largo de un gradiente latitudinal, basados en las características morfológicas de las hojas y partiendo del supuesto de que la variación fenotípica de estos caracteres está asociada a la existencia de la variación genética. La variación morfológica también puede indicar plasticidad fenotípica, como un mecanismo para la adaptación de los organismos ante la heterogeneidad del medio, que se expresa en caracteres relacionados directamente con la adecuación y la evolución bajo condiciones ambientales particulares (Franiel y Wieski 2005, Chevin et al. 2010). Para el presente estudio, se formuló la siguiente hipótesis: existe una diferenciación significativa entre poblaciones de $P$. patula para caracteres morfológicos, a través de un gradiente altitudinal. Los objetivos del presente trabajo fueron: a) cuantificar la variación fenotípica entre poblaciones de P. patula en caracteres morfológicos (acículas, conos y semillas); b) establecer lineamientos provisionales para el movimiento de semilla a partir de la variación fenotípica de caracteres morfológicos con valor adaptativo; y c) comparar la zonificación altitudinal resultante de la variación fenotípica de caracteres morfológicos con la obtenida con base en la variación genética altitudinal en la altura de la planta evaluada en un ensayo de procedencias.

\section{MÉTODOS}

Recolecta del material biológico. Se ubicaron 13 sitios de recolecta de muestras (cuadro 1 ) en un transecto altitudinal en la distribución natural de $P$. patula en los bosques de la comunidad indígena de Ixtlán de Juárez, Oaxaca, México. El sitio de recolección se denominó como procedencia y al conjunto de árboles muestreados en cada sitio se denominó como población. Los sitios dentro del transecto estuvieron ubicados aproximadamente a $50 \mathrm{~m}$ de diferencia altitudinal, de los 2.450 a los 3.050 m s.n.m. En cada población muestreada se seleccionaron 11 árboles al azar, separados entre sí de 30 a $50 \mathrm{~m}$, libres de plagas y enfermedades, con conos maduros y cerrados. De cada individuo, se recolectaron cinco estróbilos femeninos maduros (llamados comúnmente conos) y una muestra de ramas. Las ramas se recolectaron con fascículos sin señales de decaimiento. Esta muestra y los conos obtenidos se guardaron en bolsas que permitían la transpiración, con una etiqueta exterior y una interior. Durante el muestreo se mantuvo aproximadamente una misma exposición: $35^{\circ} \mathrm{NE}$. Posteriormente, para su medición se trasladó el material a las instalaciones del Instituto de Investigaciones Agropecuarias y Forestales de la Universidad Michoacana de San Nicolás de Hidalgo en Morelia, Michoacán, México. 
Cuadro 1. Localización geográfica y altitudinal de 13 poblaciones de Pinus patula var. patula muestreadas a lo largo de un transecto altitudinal en Ixtlán de Juárez, Oaxaca, México.

Geographical and altitudinal location of 13 Pinus patula. var. patula populations sampled along an altitudinal transect at Ixtlán de Juárez, Oaxaca, Mexico.

\begin{tabular}{|c|c|c|c|}
\hline Población & Latitud N & Longitud O & Altitud (m s.n.m.) \\
\hline 1 & $17^{\circ} 22,669^{\prime}$ & $96^{\circ} 26,920^{\prime}$ & 3.050 \\
\hline 2 & $17^{\circ} 22,270^{\prime}$ & $96^{\circ} 26,987^{\prime}$ & 3.000 \\
\hline 3 & $17^{\circ} 23,154^{\prime}$ & $96^{\circ} 27,402^{\prime}$ & 2.950 \\
\hline 4 & $17^{\circ} 23,185^{\prime}$ & $96^{\circ} 27,839^{\prime}$ & 2.900 \\
\hline 5 & $17^{\circ} 21,793^{\prime}$ & $96^{\circ} 27,399$ ' & 2.850 \\
\hline 6 & $17^{\circ} 21,795^{\prime}$ & $96^{\circ} 27,562^{\prime}$ & 2.800 \\
\hline 7 & $17^{\circ} 23,004^{\prime}$ & $96^{\circ} 28,577^{\prime}$ & 2.750 \\
\hline 8 & $17^{\circ} 21,749^{\prime}$ & $96^{\circ} 27,880^{\prime}$ & 2.700 \\
\hline 9 & $17^{\circ} 23,162^{\prime}$ & $96^{\circ} 28,938^{\prime}$ & 2.650 \\
\hline 10 & $17^{\circ} 23,059^{\prime}$ & $96^{\circ} 28,861^{\prime}$ & 2.600 \\
\hline 11 & $17^{\circ} 22,902^{\prime}$ & $96^{\circ} 28,902^{\prime}$ & 2.550 \\
\hline 12 & $17^{\circ} 22,808^{\prime}$ & $96^{\circ} 29,067^{\prime}$ & 2.500 \\
\hline 13 & $17^{\circ} 22,679^{\prime}$ & $96^{\circ} 28,986^{\prime}$ & 2.450 \\
\hline
\end{tabular}

Mediciones morfológicas. De la muestra botánica de ramas con fascículos, se tomó una muestra de dos fascículos de al menos un año de edad (para asegurar que hubieran completado su crecimiento) por individuo, y se midió el largo de la acícula más larga (cm) de cada uno de los fascículos, y el largo de la vaina (mm) del mismo.

Cuando los conos aún estaban cerrados, se determinó la longitud $(\mathrm{cm})$ y grueso $(\mathrm{cm})$ de éstos. La longitud del cono se midió del pedúnculo a la punta del cono, y el grueso en la parte media del mismo. Posteriormente, los conos se colocaron en contenedores por separado por cada árbol, y se expusieron al sol; una vez abiertos se extrajo las semillas. De dos escamas de la parte central y exterior del cono se evaluó el ancho de quilla (mm) y el largo de apófisis (mm). El ancho de quilla se midió en la parte transversal media de la escama, y el largo de apófisis desde la base hasta donde se inicia la espina de la escama.

A una muestra de dos semillas por individuo de cada población se les midió el largo (mm) y el ancho (mm). El largo de la semilla se determinó desde la base hasta la punta de la semilla, y el ancho en la parte más amplia de la misma. Así mismo, se midió el largo (mm) y ancho del ala de la semilla (mm). El largo del ala de la semilla se midió desde la base hasta el ápice del ala, y el ancho en la parte más amplia de la misma.

Para la evaluación, se eligieron una serie de caracteres morfológicos de fácil medición, que pueden ser obtenidos mediante una simple colecta de ramas y conos de individuos creciendo en poblaciones naturales. Algunos caracteres pueden tener valor adaptativo (como la longitud de acículas), pero se asume que la variación entre poblaciones de otros caracteres morfológicos sin valor adaptativo conocido (de uso común en taxonomía clásica, como ancho de quilla de las escamas) pueden servir como un indicador indirecto de la posible variación genética entre poblaciones (Roth y Mercer 2000).

Análisis estadístico. Para determinar la existencia de variación significativa entre poblaciones de las diversas características morfológicas (acículas, conos y semillas), se realizó un análisis de la varianza mediante el procedimiento GLM del paquete estadístico SAS (SAS 2004). El modelo utilizado para las características evaluadas fue:

$\mathrm{Y}_{i j}=\mu+$ Población $_{\mathrm{i}}+$ Árbol(Población $)_{i j}+\varepsilon_{i j}$

Donde: $\mathrm{Y}_{i j}=$ valor de la ij-ésima observación, $\mu$ = media general, Población $n_{i}=$ efecto fijo de la $i$-ésima población, Árbol(Población) ${ }_{i j}=$ efecto aleatorio del $j$-ésimo árbol anidado en la i-ésima población, $\varepsilon_{i j}=$ error experimental.

Se estimaron los componentes de varianza asociados a cada fuente de variación y su contribución a la varianza total, mediante el procedimiento VARCOMP, opción REML (SAS 2004).

Para determinar si existía un patrón de variación morfológica altitudinal, expresados como la asociación entre los valores medios por población de cada característica evaluada con la altitud sobre el nivel del mar de cada procedencia, se utilizó el procedimiento REG de SAS (SAS 2004) con el siguiente modelo estadístico:

$\mathrm{Y}_{i}=\mu+\beta_{0}+\beta_{1} \mathrm{X}_{i}+\varepsilon_{i}$

Donde: $\mathrm{Y}_{i}=$ valor de la $i$-ésima observación, $\mu=$ media general, $\beta_{0}=$ interceptada, $\beta_{1}=$ parámetro de regresión, $X_{i}$ $=$ altitud sobre el nivel del mar de la $i$-ésima población, $\varepsilon_{i}$ $=$ error.

Se excluyeron del análisis los valores de grueso de cono de la población de 2.900 m s.n.m. por un error originado en la captura de datos.

Zonificación altitudinal. Se seleccionó el largo de la acícula más larga para realizar la zonificación altitudinal. Esta característica presentó asociación significativa con la altitud sobre el nivel mar, y probablemente es la de mayor importancia fisiológica y adaptativa, al estar directamente relacionada con los procesos de fotosíntesis y transpiración de las plantas. Las hojas cumplen un papel importante en la supervivencia y crecimiento de las plantas por la adquisición de los recursos disponibles en su ambiente, y se adaptan a diferentes condiciones ambientales, y reaccionan sensiblemente a éstas (Xu et al. 2008); las hojas son uno de los órganos que más varía con la altitud sobre el nivel del mar (Niniz-Romero 2005, Zúñiga et al. 2009) o con la latitud (Uribe-Salas et al. 2008). La relevancia de lo anterior reside en el supuesto de que la variación en caracteres morfométricos funcionales que están directamente ligados al 
desarrollo de los organismos, y puede ser un indicador de la variación genética cuantitativa de la especie como una respuesta a la selección natural (Roth y Mercer 2000). Se estimó la diferencia mínima significativa (LSD) entre poblaciones del largo de la acícula más larga con un $\alpha=0,20$, para obtener los intervalos altitudinales; cabe mencionar que el utilizar el valor de $\alpha$ antes señalado conlleva a reglas de manejo más conservadoras, debido a que los intervalos altitudinales resultantes (que hacen a dos poblaciones significativamente diferentes) es menor, y por tanto habrá más restricciones en el movimiento de la semilla (SáenzRomero et al. 2006). Valores de $\alpha=0,2$ se han usado previamente para establecer límites de las zonas altitudinales (Rehfeldt 1991, Sáenz-Romero et al. 2006, Sáenz-Romero y Tapia-Olivares 2008, Viveros-Viveros et al. 2009).

\section{RESULTADOS}

Variación morfológica. Todas las variables evaluadas mostraron variación significativa tanto entre poblaciones como entre árboles dentro de poblaciones $(P<0,05$, cuadro 2). La contribución de las poblaciones a la varianza total varió del 4,4 al 32,5\%, mientras que la de los árboles dentro de poblaciones fue del 16,2 al 78,5 \% (cuadro 2). Las variables morfológicas que presentaron mayor variación atribuible a las poblaciones fueron la longitud de la vaina y la longitud del cono (32,5 \% y 26,2 \% respectivamente), mientras que la de menor variación atribuible a las poblaciones fue la longitud de acícula más larga (4,4 \%). Por otra parte, la longitud de la acícula más larga y el grueso del cono fueron las que presentaron mayor variación atribuida a árboles dentro de poblaciones (78,5 \% y 66,1 $\%$, respectivamente), y la variable con menor aporte a esta fuente de variación fue el ancho del ala de la semilla 16,2
\% (cuadro 2). En todas las variables, la contribución a la varianza total fue mayor por los árboles dentro de poblaciones que por las mismas (cuadro 2).

Patrones altitudinales de variación morfológica. Se encontraron dos patrones significativos de variación morfológica altitudinal. Por un lado, la correlación entre la media de la población y la altitud de origen de las poblaciones fue positiva para el ancho de quilla $(r=0,67 ; P=0,0119)$, el grueso del cono $(r=0,75 ; P=0,0046)$ y la longitud de la vaina $(r=0,83 ; P=0,0005)$; es decir, las poblaciones de mayor altitud presentaron conos más gruesos, las escamas de los mismos presentaron quillas más anchas y fascículos con vainas más largas, mientras que las poblaciones de menor altitud mostraron conos menos gruesos, con escamas con quillas más angostas y fascículos con vainas más cortas (figura 1A, 1B y 1C). En contraste, la longitud de acícula mostró una asociación negativa con la altitud ( $r=-0,59 ; P=0,032)$, lo que indica que las poblaciones de mayor altitud mostraron acículas más cortas que las poblaciones de menor altitud (figura 1D). La estimación de la pendiente derivado del ajuste del modelo de regresión lineal entre la longitud de acícula más larga y la altitud de origen de las poblaciones, indica que por cada $100 \mathrm{~m}$ que se ascienda sobre el nivel del mar, la longitud de las acículas disminuirá aproximadamente $3 \mathrm{~mm}\left(\mathrm{r}^{2}=0,35\right.$; $P=0,032$; figura 1D).

Zonificación altitudinal con base en la longitud de acícula. Con base en la diferencia mínima significativa para la longitud de acícula (DMS ${ }_{\alpha=0,2}=1,66 \mathrm{~cm}$ ) se delimitaron dos zonas altitudinales para el movimiento de semillas, que cubren la totalidad del área de distribución altitudinal de la especie en la región. Cada zona tuvo una amplitud de $450 \mathrm{~m}$

Cuadro 2. Media, error estándar, porcentaje de contribución a la varianza total (\%) y significancia $(P)$ de características morfológicas para 13 poblaciones de Pinus patula var. patula en Ixtlán de Juárez, Oaxaca, México.

Mean, standard error, contribution to total variance (\%) and significance (P) of morphological traits from 13 Pinus patula var. patula populations at Ixtlán de Juárez, Oaxaca, Mexico.

\begin{tabular}{|c|c|c|c|c|c|c|c|}
\hline \multirow[t]{2}{*}{ Variable } & \multirow[t]{2}{*}{ Media } & \multirow{2}{*}{$\begin{array}{c}\text { Error } \\
\text { estándar }\end{array}$} & \multicolumn{2}{|c|}{ Población } & \multicolumn{2}{|c|}{ Árbol (población) } & \multirow{2}{*}{$\begin{array}{c}\text { Error } \\
\% \\
\end{array}$} \\
\hline & & & $\%$ & $P$ & $\%$ & $P$ & \\
\hline Longitud de acícula más larga (cm) & 19,8 & 0,19 & 4,4 & $<0,0001$ & 78,5 & $<0,0001$ & 17,1 \\
\hline Longitud de vaina (mm) & 14,4 & 0,19 & 32,5 & $<0,0001$ & 46,0 & $<0,0001$ & 21,5 \\
\hline Longitud de cono (cm) & 5,8 & 0,07 & 26,2 & $<0,0001$ & 39,9 & $<0,0001$ & 33,9 \\
\hline Grueso de cono (cm) & 2,7 & 0,03 & 11,4 & $<0,0001$ & 66,1 & $<0,0001$ & 22,5 \\
\hline Largo de apófisis (mm) & 2,9 & 0,04 & 11,5 & $<0,0001$ & 51,5 & $<0,0001$ & 37,0 \\
\hline Ancho de quilla (mm) & 10,2 & 0,10 & 14,8 & $<0,0001$ & 43,1 & $<0,0001$ & 42,1 \\
\hline Largo de semilla (mm) & 5,2 & 0,05 & 9,0 & $<0,0001$ & 54,4 & $<0,0001$ & 36,6 \\
\hline Ancho de semilla (mm) & 2,6 & 0,02 & 15,1 & $<0,0001$ & 40,6 & $<0,0001$ & 44,3 \\
\hline Largo del ala de la semilla (mm) & 15,0 & 0,17 & 17,9 & $<0,0001$ & 51,2 & $<0,0001$ & 30,9 \\
\hline Ancho del ala de la semilla (mm) & 5,0 & 0,05 & 10,0 & 0,0004 & 16,2 & 0,04470 & 73,8 \\
\hline Promedio & ------ & ---- & 15,3 & - ---- & 48,7 & ----- & 36,0 \\
\hline
\end{tabular}



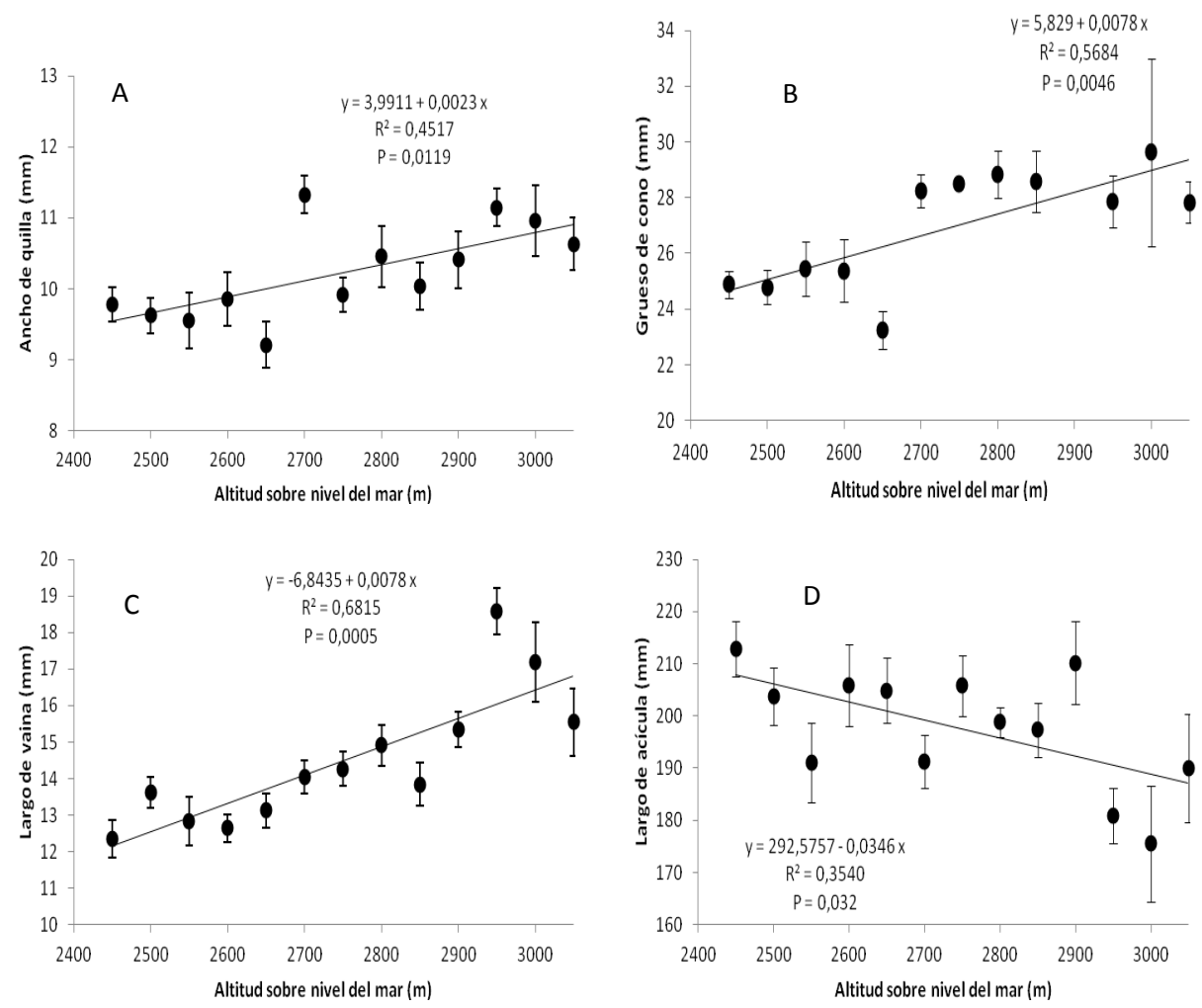

Figura 1. Relación entre las variables morfológicas: A) ancho de quilla, B) grueso de cono, C) largo de vaina y D) largo de acícula, con la altitud de origen de 13 poblaciones de Pinus patula var. patula en Ixtlán de Juárez, Oaxaca, México. Las barras verticales representan el error estándar.

Relationship among the morphological traits: a) keel width, b) cone width, c) leaf-sheaths length and d) needle length and the altitude of origin of 13 Pinus patula var. patula populations. Vertical bars represent the standard error.

de diferencia altitudinal. La primera zona (parte baja de la distribución natural) se ubicó entre 2.150 y 2.600 m s.n.m., y la segunda zona entre 2.600 y 3.050 m s.n.m. (parte alta de la distribución natural). Por lo que se sugiere que la semilla recolectada en cada zona se utilice de manera provisional en reforestaciones dentro del intervalo altitudinal, o bien se pueda mover $\pm 225 \mathrm{~m}$ altitudinales a partir del lugar de cosecha. Esto sin considerar los efectos del cambio climático.

\section{DISCUSIÓN}

Variación morfológica. La diferenciación significativa mostrada por las características morfológicas tanto a nivel de poblaciones como a nivel de árboles dentro de poblaciones, indica que estas características están influenciadas por el ambiente y que probablemente también exista diferenciación genética entre poblaciones, sin que sea posible separar el efecto ambiental del genético en el presente trabajo. Los resultados coinciden con lo encontrado en otras especies de coníferas (Bermejo-Velázquez y Patiño-Valera 1982, Reyes-Hernández et al. 2005), pero difiere en parte a lo encontrado por Pérez-Rodríguez y Eguiluz-Piedra (1985), quienes reportan variación significativa sólo entre árboles dentro de poblaciones, pero no entre poblaciones, para las características de los conos en Pinus hartwegii Lindl. También difieren de lo encontrado por Dangasuk y Panetsos (2004), quienes reportan que la longitud de cono sólo mostró variación significativa entre poblaciones en Pinus brutia Ten, pero no variación significativa entre árboles. Estos dos últimos trabajos ubicados también en transectos altitudinales.

La elevada contribución a la varianza aportada por los árboles dentro de poblaciones concuerda con los resultados reportados en la mayoría de los estudios realizados en otras especies arbóreas, principalmente pinos (Bermejo-Velázquez y Patiño-Valera 1982, Pérez-Rodríguez y Eguiluz-Piedra 1985, Uribe-Salas et al. 2008). Por otra parte, Prieto (1992) en el género Picea y Reyes-Hernández et al. (2005) en el género Pseudotsuga encontraron que la mayor aportación a la varianza total estuvo dada por las poblaciones, en lugar de los árboles dentro de poblaciones; lo anterior, tal vez debido a que estos dos géneros presentan una distribución geográfica fragmentada y discontinua en México, lo que acrecentó la diferenciación de las poblaciones. Bermejo-Velázquez y Patiño-Valera (1982) reportan valores muy cercanos a los encontrados en este trabajo en cuanto a la contribución a la varianza total por las poblaciones y por los árboles dentro de poblaciones para 
la longitud de acículas en Pinus oaxacana Mirov. (Pinus pseudostrobus var. oaxacana Mtz.).

Patrones altitudinales de variación morfológica. La correlación positiva significativa existente entre la longitud de vaina y el grueso de cono con la altitud de origen de las poblaciones, llevan a plantear la pregunta de si esos caracteres podrían tener un papel adaptativo. Por ejemplo, Niembro (1986) y Silva et al. (2011) mencionan la hipótesis de que las vainas pueden cumplir funciones de protección a las acículas contra factores ambientales, dentro de los cuales se puede considerar a las heladas; esta suposición se basa en que se encontró en promedio que las vainas son más largas a mayores altitudes. En este mismo sentido, también podría plantearse la hipótesis de que la presencia de conos más gruesos pudiera brindar una mayor protección de las semillas contra daño por heladas, ya que estos mismos autores mencionan que los conos cumplen funciones de protección a la semilla. Por otra parte, la asociación negativa significativa entre la longitud de acícula y la altitud de origen de las poblaciones, se debe a que los árboles reducen el tamaño de sus acículas conforme se ubican en sitios con mayor elevación, para reducir el área foliar expuesta a temperaturas más bajas; o bien simplemente a un efecto de plasticidad fenotípica. Este patrón altitudinal coincide con lo reportado por Niniz-Romero (2005), quién encontró una correlación negativa significativa entre la longitud de acícula más larga y la altitud de origen de las poblaciones en Pinus hartwegii Lindl. ( $\left.r^{2}=0,80 ; P=0,0001\right)$.

Zonificación altitudinal. Con propósitos de reforestación y de restauración, se recomienda utilizar la semilla dentro de la misma zona donde fue recolectada, o bien se puede mover $\pm 225 \mathrm{~m}$ altitudinales a partir del lugar de colecta, es decir, la mitad de la amplitud de cada zona altitudinal (450 m). Esto sin considerar medidas de adaptación al cambio climático. En ese caso, probablemente sería necesario subir en elevación el ancho máximo de la zona (450 m), a partir del sitio de colecta, para reacoplarse al clima para el cual están adaptadas las poblaciones, pero que ocurrirá a mayor altitud en el futuro (Sáenz-Romero et al. 2010).

Ruiz-Talonia (2010) realizó una zonificación altitudinal en Pinus patula en la misma zona de Ixtlán basándose en la variación genética altitudinal del crecimiento en altura de la planta a los 24 meses de edad detectada en un ensayo de procedencias en campo, y delimitó tres zonas para el movimiento de semilla, la primera entre los 2.400 y 2.600 m s.n.m., la segunda entre los 2.600 y 2.800 m s.n.m. y la tercera entre los 2.800 y los 3.000 m s.n.m.; cada zona altitudinal con una amplitud de $200 \mathrm{~m}$ altitudinales y con un intervalo para el movimiento de semillas de $\pm 100 \mathrm{~m}$ altitudinales, con un $\alpha=0,20$.

Al comparar la zonificación obtenida de la variación en la longitud de acículas con la zonificación resultante de la variación genética altitudinal del crecimiento en altura de la planta a los 24 meses de edad, a partir de ensayos de procedencias en campo (Ruiz-Talonia 2010), se observa que esta última comprende más zonas con menor amplitud altitudinal y por lo tanto, un intervalo menor para el movimiento de semillas. Esto conduce a un movimiento de semillas más conservador.

La relevancia de la evaluación de la variación de algunos caracteres morfológicos con valor adaptativo es que a partir de esta, se puede generar una zonificación preliminar para el movimiento de semillas, en este caso con un resultado menos conservador o más liberal, al que se obtiene con el estudio de la variación de caracteres cuantitativos en ensayos de procedencias en campo, pero sin el costo que implica establecer, mantener y evaluar los ensayos de procedencias. Es decir, la zonificación con base en caracteres morfológicos de valor adaptativo, sería una alternativa económica, si bien no ideal, para obtener una zonificación altitudinal provisional. Un ejemplo de ello es también el trabajo de Uribe-Salas et al. (2008) con Quercus rugosa.

Se sugiere realizar más comparaciones de zonificaciones con base en caracteres morfológicos que tengan valor adaptativo con las zonificaciones obtenidas de la variación genética altitudinal de caracteres cuantitativos, para las especies que ya cuenten con esta última.

\section{CONCLUSIONES}

La variación de la longitud de acículas caracteres morfológicos entre y dentro de poblaciones, permite obtener una zonificación altitudinal para el movimiento de semillas, similar a la obtenida con la variación de caracteres cuantitativos en ensayos de campo. Las zonas altitudinales obtenidas a partir de la variación altitudinal en la longitud de acícula fueron más amplias; es decir, son menos restrictivas que las zonas resultantes al utilizar el patrón altitudinal en el crecimiento en altura de la planta. Por ello, la zonificación altitudinal basada en caracteres morfológicos con valor adaptativo debe de tener un carácter provisional. Su implementación se recomienda en especies en las que aún no se han establecido ensayos de procedencias para evaluar la variación genética altitudinal de caracteres cuantitativos.

\section{REFERENCIAS}

Bermejo-Velázquez B, F Patiño-Valera. 1982. Variación morfológica en características de hojas y conos de Pinus pseudostrobus var. oaxacana Mtz. en poblaciones naturales de los altos de Chiapas. México, D.F. Instituto Nacional de Investigaciones Forestales. $47 \mathrm{p}$.

Chevin LM, R Lande, GM Mace. 2010. Adaptation, plasticity, and extinction in a changing environment: towards a predictive theory. PLoS Biology 8(4): 1-8.

Dangasuk OG, KP Panetsos. 2004. Altitudinal and longitudinal variation in Pinus brutia (Ten.) of Crete Island, Greece: some needle, cone and seed traits under natural habitats. New Forest 27: 269-284.

Franiel I, K Wieski. 2005. Leaf features of silver birch (Betula pendula Roth). Variability within and between two popula- 
tions (uncontaminated vs Pb-contaminated and Zn-contaminated site). Trees 19: 81-88.

Niembro R A. 1986. Mecanismo de Reproducción Sexual en Pinos. México, D.F. Limusa. 130 p.

Niniz-Romero R. 2005. Variación morfológica de conos y semillas de Pinus hartwegii Lindley, a lo largo de un transecto altitudinal en Pico de Tancítaro, Michoacán, México. Tesis Licenciatura en Biología. Morelia, Michoacán. Universidad Michoacana de San Nicolás de Hidalgo. 45 p.

Nyoka, BI. 2002. Pinus patula Schiede ex Schltdl. \& Cham. En: CAB International (comp). Pines of Silvicultural Importance. London, UK. CABI Publising. p. 303-316.

Pérez-Rodríguez PM, T Eguiluz-Piedra. 1985. Variación morfológica en Pinus hartwegii del eje Neovolcánico. In SARH (Secretaria de Agricultura y Recursos Hidráulicos). III Reunión Nacional sobre Plantaciones Forestales. México, D.F. p. 245-270. (Publicación Especial $\mathrm{N}^{\circ} 48$ ).

Prieto ME. 1992. Variación morfológica en conos y semillas de 13 poblaciones de naturales de Picea (Pinaceae) en México. Tesis Ingeniero Agrónomo especialista en Bosques. Chapingo, México. División de Ciencias Forestales, Universidad Autónoma Chapingo. 73 p.

Rehfeldt GE. 1991. A model of genetic variation for Pinus ponderosa in the Inland Northwest (USA): applications in gene resource management. Canadian Journal of Forest Research 21: 1491-1500.

Rehfeldt GE, RA Wykoff, CC Ying. 2001. Physiologic plasticity, evolution, and impacts of a changing climate in Pinus contorta. Climatic Change 50: 355-376.

Reyes-Hernández VJ, JJ Vargas-Hernández, J López-Upton, H Vaquera-Huerta. 2005. Variación morfológica y anatómica en poblaciones mexicanas de Pseudotsuga (Pinaceae). Acta Botánica Mexicana 70: 47-67.

Roth L, JM Mercer. 2000. Morphometrics in development and evolution. American Zoologist 40: 801-810.

Ruiz-Talonia LF. 2010. Variación genética altitudinal entre procedencias de Pinus patula Schltdl et Cham. en ensayos de vivero y campo en Ixtlán de Juárez, Oaxaca. Tesis Maestría en Ciencias Biológicas. Morelia, Michoacán, México. Universidad Michoacana de San Nicolás Hidalgo. 64 p.

SAS Institute. 2004. SAS/STAT 9.1 User's Guide. N.C., USA. SAS Institute Inc. 4975 p.

Sáenz-Romero C. 2004. Zonificación estatal y altitudinal para la colecta y movimiento de semillas de coníferas en México. In Vargas-Hernández JJ, B Bermejo-Velázquez, FT Ledig eds. Manejo de recursos genéticos forestales. México, D.F. CONAFOR-Comisión Forestal de América del Norte. p. 72-86.

Sáenz-Romero C, RR Gúzman-Reyna, GR Rehfeldt. 2006. Alti- tudinal genetic variation among Pinus oocarpa populations in Michoacán, México. Implications for seed zoning, conservation, tree breeding and global warming. Forest Ecology and Management 229: 340-350.

Sáenz-Romero C, BL Tapia-Olivares. 2008. Genetic variation in frost damage and seed zone delineation within an altitudinal transect of Pinus devoniana (P. michoacana) in Mexico. Silvae Genetica 57(3): 165-170.

Sáenz-Romero C, GR Rehfeldt, NL Crookston, P Duval, R StAmant, J Beaulieu, BA Richardson. 2010. Spline models of contemporary, 2030, 2060 and 2090 climates of Mexico and their use in understanding climate-change impacts on the vegetation. Climatic Change 102: 595-623.

Sáenz-Romero C, GR Rehfeldt, JC Soto-Correa, S Aguilar-Aguilar, V Zamarripa-Morales, J López-Upton. 2012. Altitudinal genetic variation among Pinus pseudostrobus populations from Michoacán, México. Two location shadehouse test results. Revista Fitotecnia Mexicana 35(2): 11-120.

Silva SP, JM Ortega R, JC Montero C, F Hernández V, JS Robles del V, G Rodríguez L. 2011. Manual de Prácticas de Laboratorio de Botánica II. Ciclo 2011. Pteridofitas y Gimnospermas. Morelia, Michoacán, México. Facultad de Biología. Universidad Michoacana de San Nicolás de Hidalgo. $118 \mathrm{p}$.

Styles BT. 1993. The genus Pinus: a Mexico purview. In Ramamoorthy TP, R Bye, A Lat, J Fa eds. Biological Diversity of Mexico: Origins and Distribution. New York, USA. Oxford Univ. Press. p. 397-420.

Uribe-Salas D, C Sáenz-Romero, A González-Rodríguez, O Telléz-Valdéz, K Oyama. 2008. Foliar morphological variation in the white oak Quercus rugosa Née (Fagaceae) along a latitudinal gradient in Mexico: potential implications for management and conservation. Forest Ecology and Management 256: 2121-2126.

Viveros-Viveros H, C Sáenz-Romero, JJ Vargas-Hernández, J López-Upton, G Ramírez-Valverde, A Santacruz-Varela. 2009. Altitudinal genetic variation in Pinus hartwegii Lindl. I: Height growth, shoot phenology, and frost damage in seedlings. Forest Ecology and Management 257: 836842.

Xu F, W Guo, G Xu, R Wang. 2008. Habitat effects on leaf morphological plasticity in Quercus acutissima. Acta Biologica Cracoviensia 50(2): 19-26.

Zúñiga EÁ, A Sánchez-González, SD Granados. 2009. Análisis de la variación morfológica foliar en Quercus laeta Liebm. en el parque nacional Los Mármoles, Hidalgo, México. Revista Chapingo Serie Ciencias Forestales y del Ambiente 15(2): 87-93. 
